\title{
Clinical Feature Classification for Chronic Heart Failure and Construction of a Safe Mechanism for Rehabilitation using Internet-of- Medical-Thing Devices
}

\section{Shao-Jie Hsu, Shih-Syun Lin, Tun-Wen Pai}

Department of Computer Science and Engineering, National Taiwan Ocean University, No. 2, Peining Rd., Keelung 202, Taiwan

e-mail: twp@mail.ntou.edu.tw

\section{Chao-Hung Wang, Min-Hui Liu}

Heart Failure Research Center, Division of Cardiology, Department of Internal Medicine, Chang Gung Memorial Hospital (Keelung), No. 222, Maijin Rd., Keelung 204, Taiwan

e-mail: bearty@cgmh.org.tw

\section{Chi-Wen Cheng}

Division of Cardiology, Department of Internal Medicine, Chang Gung Memorial Hospital (Keelung), No. 222, Maijin Rd., Keelung 204, Taiwan e-mail: chyiwen@cgmh.org.tw

\section{Dong-Yi Chen}

Division of Cardiology, Department of Internal Medicine, Chang Gung Memorial Hospital (Linkou), No. 5, Fuxing St., Taoyuan 333, Taiwan e-mail: dongyi70@cgmh.org.tw

\section{Kuo-Li Pan}

Division of Cardiology, Department of Internal Medicine. Chang Gung Memorial Hospital (Chiayi), No. 6, Sec. West, Jiapu Rd., Puzih City, Chiayi 613, Taiwan e-mail: q12070@cgmh.org.tw 


\title{
Shyh-Ming Chen
}

Division of Cardiology, Department of Internal Medicine, Chang Gung Memorial Hospital (Kaohsiung), No. 123, Dapi Rd., Kaohsiung 833, Taiwan e-mail: syming99@cgmh.org.tw

\begin{abstract}
Heart failure (HF) is a complex syndrome without an objective definition. It has become a serious problem in public health policies because of the increased prevalence, high cost of treatment, frequent re-hospitalization and high mortality. Neither strict standards for $\mathrm{HF}$ classification nor single-type treatments are currently available. The non-specific clinical symptoms make diagnosis at early stages difficult, leading to deterioration and hospitalization. The use of advanced medical techniques and newly developed medicines may decrease mortality, but many HF patients still have a low quality of life because of insufficient muscular endurance and limited activities. Recent reports have shown that exercise programs contribute to the recovery of cardiac functions and improve clinical results for most HF patients. However, excessive, intense exercise may increase the risk of death, particularly for cardiac-related patients. In this study, different HF types are categorized and a safe, customized mechanism for self-exercise training integrating Internet-of-Medical-Thing devices and cloud computing technologies is proposed. The detected biometric features of the HF patients are linked to the personal communication devices of the patients and doctors, a cloud server system and the hospital medical information system. The proposed system mainly collects heart rate and metabolic equivalent features in a real-time manner from the Internet-of-Medical-Thing devices worn by patients. Measured data are dynamically compared to customized maximum limitations that are defined by rehabilitation physicians according to the patient's cardio-pulmonary exercise testing record in the hospital. A prototype system was successfully developed and validated with several test cases and showed excellent performance at an affordable cost. The proposed mechanism provides a customized platform for HF patients to pursue a better quality of life, based on prognostic exercise prescription using a safe self-exercise training mechanism.
\end{abstract}

Keywords: chronic heart failure; ejection fraction; heart rate; metabolic equivalent; Internet-of-Medical-Thing

\section{Introduction}

\subsection{Epidemiology and Symptom of Heart Failure}

Heart failure (HF) is a complex, irreversible, costly, and high-mortality disease that is becoming an increasing health problem affecting public health policies worldwide. Epidemiologic studies have described the critical social burden of HF 
because of its high prevalence, frequent rehospitalization rates, and high healthcare costs [1]. For example, more than 5 million people reportedly suffer from HF in the United States, and more than 30 billion dollars are required for medical treatment of HF patients each year [2]. The prevalence of HF in the Taiwanese population is as high as $16 \%$ for elderly people (age greater than 65 years) and annual reports of health statistics have shown that heart-related diseases have become the second leading cause of death in recent years [3]. Similar to that in other countries, the health budget burden from frequent rehospitalization caused by HF-related diagnoses is significantly increased. The cost of hospitalization for HF patients has increased by an average of $5.5 \%$ over the past decade in Taiwan. This may be because of the success of thrombolysis and intravascular stent therapies, which have greatly increased the survival rates of myocardial infarction patients in recent years. However, all heart-related diseases and most chronic diseases can progressively become an epidemic of heart failure and create a major public health problem worldwide [4]. Despite the development of advanced medications and surgical therapies for HF patients, HF remains a progressive, irreversible, and ultimately fatal disease over a short period because of poor prognosis.

The general symptoms of HF are associated with progressive conditions of fatigue and dyspnea, and limit the daily activities of patients, resulting in serious invisible disabilities [5]. However, the symptoms of HF are not obvious at earlier stages, and patients often ignore common symptoms such as asthma or breathing problems. Thus, HF patients at risk are frequently reluctant to seek medical assistance until they are in serious conditions or in a late-stage. Another challenge is that HF symptoms are quite variable, such as abnormal breathing conditions, dyspnea, fatigue, leg edema, difficulty sleeping, loss of appetite, coughing with phlegm or mucus foam, memory loss, and confusion. HF is a complex syndrome without a simple objective definition and is easily misdiagnosed like other chronic diseases, limiting the opportunity for early therapies.

\subsection{Major Categories of HF Disease}

Accurate diagnosis of $\mathrm{HF}$ enables appropriate management and greater benefit from early treatment for HF patients. In this study, we initially divided HF patients into five major groups including systolic, diastolic, valvular, atrial fibrillation, and non-specified HF types. Systolic HF is very common in male patients and those with ischemic heart diseases [6]. Clinically, we used an ejection fraction (EF) of $50 \%$ in the left ventricle as a threshold [7]. Valvular heart disease is characterized by damaged conditions or a defect in one of the four heart valves: mitral and aortic valves in the left heart and tricuspid and pulmonary valves in the right heart. Normal functioning valves ensure that blood flows with proper force, direction, and time. In valvular type heart disease, the valves become too narrow and hardened (stenotic) to open fully, or are unable to close completely (incompetent). 
A stenotic valve forces blood to flow back to the adjacent heart chamber, while an incompetent valve leaks blood back into the previous chamber [8]. To compensate for the poor pumping action, the heart muscle enlarges and thickens, thereby losing elasticity and efficiency. Diastolic HF is ascribed to the natural effect of aging on the heart. It is mainly caused by the stiffening of heart muscles, which can prevent the patient's heart from properly filling with blood [9]. Diastolic HF may not reduce the ejection fraction compared to systolic HF patients. In diastolic $\mathrm{HF}$, the left ventricle may pump well during the systole; however, it does not fill with enough blood during the diastole. A patient's ventricle may have a normal $\mathrm{EF}$, but an insufficient amount of blood is pumped out. As a result, the ventricle pumps out less blood with each beat. The criteria for the diagnosis of diastolic HF remain imprecise, making it difficult to conduct valid clinical trials of treatment. The problem is compounded by the fact that systolic and diastolic HF commonly coexist when patients present with many ischemic and non-ischemic etiologies of HF. Narrowly defined, diastolic HF has often been defined as "heart failure with normal systolic function" (i.e. LVEF is greater than 50\%) [10]. In this study, we also adopted an EF of $50 \%$ as a criterion in accordance with pulmonary edema and congestion conditions from chest X-ray examination reports to identify diastolic HF cases. Atrial fibrillation (AF) appears with abnormal heart rhythm and can be characterized and diagnosed as rapid and irregular beating through an electrocardiogram device [11]. AF is strongly associated with heart failure, coronary artery disease, valvular heart disease, diabetes mellitus, and hypertension. Normally, findings from 12-lead electrocardiogram can confirm the diagnosis of AF; hence, patients with an annotated ICD-9 code of 42731 can be directly confirmed as having AF. The remaining HF patients not satisfying the criteria described above can be categorized into a non-specified HF group, as there are many subtypes that must be further defined according to various combination syndromes.

\subsection{Cardiac Rehabilitation for HF Patients}

Once the major HF types are accurately identified, proper medications and surgical treatments can be applied. In addition to traditional therapies, exercise prescription may improve health conditions in both psychological and physiological aspects. A recent report revealed that intervened exercise training programs for HF patients achieved a 35\% reduction in the risk of death and $28 \%$ reduction for both all-cause mortality and hospitalization [12]. This additional cardiac rehabilitation can be considered as a preventive medical prescription and will reduce the symptoms of HF patients, including enhanced endothelial cell functions of blood vessels [13], improved exercise tolerance by increasing muscle oxygen uptake efficiency [14], reducing sympathetic nerve activities [15], and reducing inflammation [16]. It is also recommended by the American Heart Association and European Society of Cardiology that all stabilized HF patients should be advised to participate in exercise training therapies [5]. 
However, clinical trials of exercise intensity, frequency, duration, type of exercise equipment, and exercise protocols should be customized for each HF patient, based on cardio-pulmonary exercise testing (CPX) assessments [17]. CPX is a common and noninvasive measuring procedure used in clinical practice which to provide precise and reliable assessment of various factors in response to maximal aerobic capacity including cardiac and pulmonary measurements. As customized maximal fitness levels are instructed by rehabilitation physicians, long-term and self-training activities can be performed at home according to the exercise prescription. Although regular exercise can help to improve survival rates of $\mathrm{HF}$ patients, high-intensity exercises may also increase the risk of sudden death. Hence, a proper or safe mechanism for self-training at home is very important for HF patients.

\subsection{Internet of Medical Things and Two Adopted Biometric Features}

Internet of Things (IoT) is a network connecting various physical devices that allow objects to collect or exchange data from each other. Among the IoT application areas, the medical care application referred to as the Internet of Medical Things (IoMT) has gained attention and can be constructed by connecting healthcare wearable devices, enabling doctors to remotely supervise and diagnose patient conditions [18]. In this study, we adopted bracelet devices and propose a safe mechanism for HF patient self-training programs. By wearing a bracelet and collecting both heart rate (HR) and metabolic equivalent (MET) data, this system can automatically send an warning message to patients, doctors, and healthcare assistants simultaneously if a dangerous event occurs. In addition, the system can detect whether the user is at risk of HF symptoms by comparing her/his biometric values with those of normal healthy people. Once the user is identified as a potential HF patient, the system offers a pre-registration function of instant notification message to the users. Particularly, when a user is at a critical status according to the collected health bracelet data, the developed system will immediately send out urgent notifications to doctors, family, or caregivers to prevent dangerous consequences.

When patients are diagnosed with HF and categorized as having a specific type of $\mathrm{HF}$, doctors may advise patients to participate in rehabilitation training to recover cardiac function. However, in order to prevent over-exercising, rehabilitation professionals perform the CPX test for individual evaluation of exercise intensity limits. Two major biometric features are applied in our developed system, including peak oxygen uptake $\left(\mathrm{VO}_{2} \max \right)$ and heart rate (HR). $\mathrm{VO}_{2} \max$ can be transferred to corresponding metabolic equivalent (MET) by dividing by 3.5 and patient weight $(\mathrm{kg})$. One unit of MET is defined as an energy consumption of 3.5 $\mathrm{mL}$ oxygen per kilogram per minute or defined as the rate of energy produced per unit surface area of an average person seated at rest [19]. Different exercise 
intensities reflect different MET values. For example, the physical activity of walking at a general speed is approximately equal to an MET value of 3.6 and jogging activity is close to an MET value of 7 . Thus, higher METs indicate greater exhaustion. In this designed system, the maximum limitations for endurance or resistance trainings for $\mathrm{HF}$ patients were initially set as either $40-80 \%$ of maximum MET $\left(\mathrm{Vo}_{2} \mathrm{max} /\right.$ weight/3.5) or maximum predicted HR (220 minus age in years). When either condition is exceeded, the warming mechanism will be activated for safety monitoring.

This paper is organized as follows. Section 2 describes the procedures of feature extraction from medical documents and corresponding statistics. Section 3 introduces a newly proposed safe mechanism for self-exercise training program and all module functions, and Sections 4 presents our conclusions.

\section{Medical Records and Feature Extraction}

\subsection{Data Sources and Feature Extraction}

This study analyzed a total of 103,114 HF patients collected from four divisions of Chang Gung Memorial Hospital (CGMH) in Taiwan (IRB:105-0504C), including the Keelung (22,664), Linkou (42,052), Chiayi $(15,662)$, and Kaohsiung $(22,736)$ divisions. At least one hospitalization or outpatient medical record annotated with an ICD-9 of 428 or 42731 is required, and both ultrasound (M22) and chest X-ray (75-011) examination reports for each HF patient from years 2004 to 2013 are basic requirements for HF type categorization. Under such limitations, only 62,159 patients remained for classification in this study, including Keelung $(16,694)$, Linkou $(13,649)$, Chiayi $(13,474)$, and Kaohsiung (18,342). According to the general rules shown in Table 1, we divided the HF patients into five major categories including systolic, diastolic, valvular, AF, and non-specified types. After the data screening processes, only $42 \%$ of HF patients could be primarily classified into the first four types including systolic, diastolic, valvular, or AF categories. Based on this low percentage, HF is indeed, a complex disease with complicated symptoms. More than $50 \%$ of $\mathrm{HF}$ patients require additional criteria to specifically identify the myocardial abnormalities associated with valve, pericardium, endocardium, heart rhythm and conduction issues. 
Table 1

Criteria for classified HF types

\begin{tabular}{|c|l|}
\hline HF type & Classified rule \\
\hline Systolic & EF lower than 50\% \\
\hline Valvular & $\begin{array}{l}\text { Mitral Regurgitation / Mitral Stenosis } \\
\text { / Aortic Regurgitation / Aortic } \\
\text { Stenosis been classified as moderate } \\
\text { to severe or SEVERE }\end{array}$ \\
\hline Diastolic & $\begin{array}{l}\text { EF higher than 50\% and reported } \\
\text { edema or congestion in patient's } \\
\text { Chest X-rays examination report }\end{array}$ \\
\hline $\begin{array}{c}\text { Atrial } \\
\text { fibrillationC\{PX }\end{array}$ & Annotated with ICD-9 code of 42731 \\
\hline Non-specified & $\begin{array}{l}\text { Not been classified as systolic, } \\
\text { valvular, diastolic type }\end{array}$ \\
\hline
\end{tabular}

\subsection{Data Extraction Method}

Regular expression was used to extract features from all of the collected clinical documents. To evaluate ultrasound test reports, we applied regular expression to examine all associated features, including patient EF values, valvular conditions (mitral stenosis, mitral regurgitation, aortic stenosis, aortic regurgitation), left ventricular end-diastolic dimension, left ventricular end-systolic dimension, interventricular septal end diastolic dimension, left ventricular end diastolic posterior wall dimension, aortic root, left atrial dimension, the ratio of early transmittal flow velocity and early mitral annular velocity. In addition, the system analyzed reports of patient chest X-rays examination simultaneously to determine whether the keywords of "edema" or "congestion" conditions were annotated within the documents. If the patient had participated in CPX testing, the $\mathrm{VO}_{2}$ max values were also retrieved to set exercise training limitations.

\subsection{Data Analysis and Statistics}

To identify additional features of HF patients, we categorized HF patient into five groups as described above and further clustered each group into several subgroups according to different genders and age intervals. The experiments were performed by analyzing hospitalization medical documents for HF patients within different age intervals. Statistical analysis showed that male patients in systolic, diastolic, valvular, and AF types were 2, 4, 6, and 4 years younger than female patients, respectively. However, this was not obvious for the cluster of non-specified HF patients. Additionally, the average age of hospitalization of valvular and diastolic 
HF patients was higher than for other subgroups. In Figure 1, the Venn diagram shows the relationship between clustered patients for the five HF types in CGMH. Based on the defined criteria, there was some overlap between populations with different comorbidities among diastolic, valvular, systolic, and AF types. The largest HF group in CGMH was the systolic type. However, the non-specified HF population accounted for more than $50 \%$ of patients; the number of non-canonical forms of diastolic HF is increasing because of the aging society in most developed countries. More diastolic HF subtypes are expected to be defined and categorized in the near future.

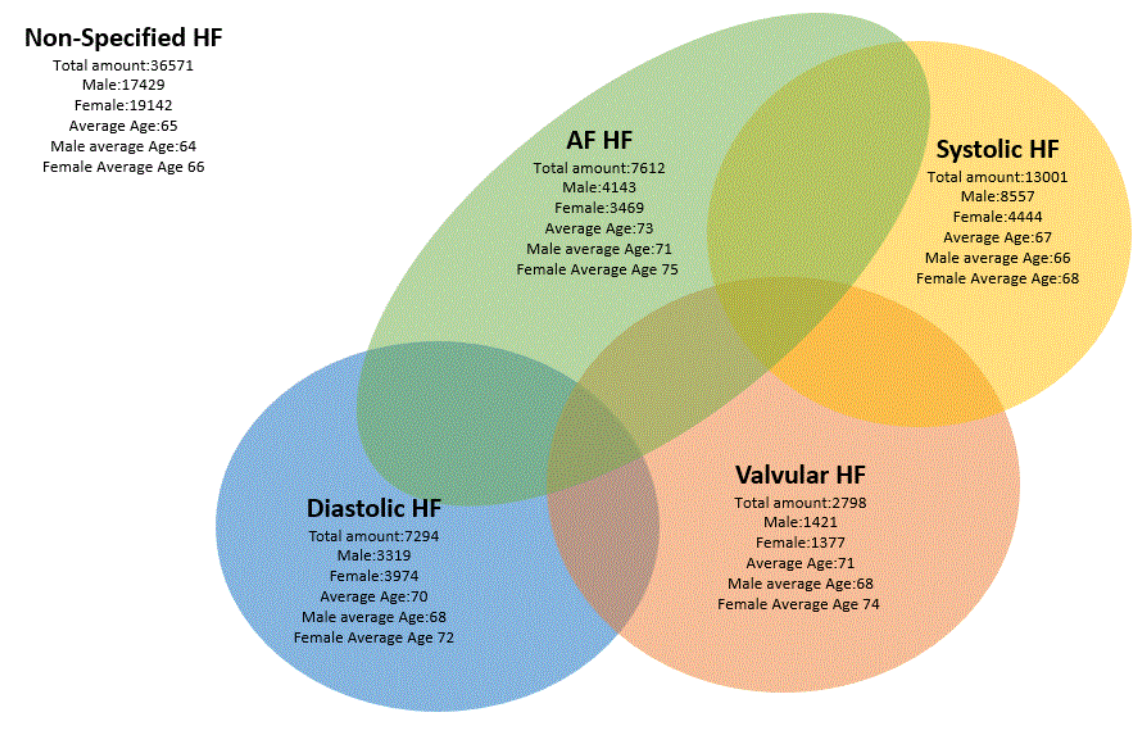

Figure 1

Venn diagram of number statistics of each HF type in four divisions of CGMH, Taiwan

\section{A Safe Mechanism Design for Self-Exercise Training}

\subsection{System Mechanism}

The proposed mechanism is divided into three modules. The first module includes a raw medical database analytical server and medical information systems. The raw medical database of HF patients from CGMH and healthy people were collected and analyzed for reference clusters. The server side connects with the hospital information systems and provides emergency messages to doctors as well 
as providing exercise prescriptions including MET and corresponding HR limitations for patients. The second module is designed for end users. After downloading the application (APP), users are required to provide personal information such as gender, age, and weight for the default settings designed in the next module. After wearing a wireless health bracelet device, the system begins to detect and record the METs and HRs of users and the bracelet connects to the APP through Bluetooth. According to the IoMT mechanism, the APP transmits physical data to cloud servers each minute based on the Message Queuing Telemetry Transport messaging protocol, a lightweight protocol for connecting with remote locations through limited network bandwidth. The final module is a cloud server for managing and analyzing the transmitted data. After receiving data from the APP, the designed system constructs a personal dataset including time, heart rate, and MET values, and the registered patient's identifier and corresponding time feature are applied as the super key for storage in the cloud database for a specific user. The integrated system configuration is shown in Figure 2. The cloud server analyzes retrieved personal data and compares them with the customized prescription limitations of the maximum MET and HR thresholds. When the physical MET/HR values exceed one of the two ranging limitations $(40-80 \%$ of MET peak or maximum predicted $\mathrm{HR}=220-$ age in years [20]), the system immediately sends a warning message to notify the patients to reduce exercise intensities. When the remote healthcare system is combined with the hospital information system, doctors can remotely monitor and inspect the patient's MET/HF values by comparison with her/his CPX testing guidelines.

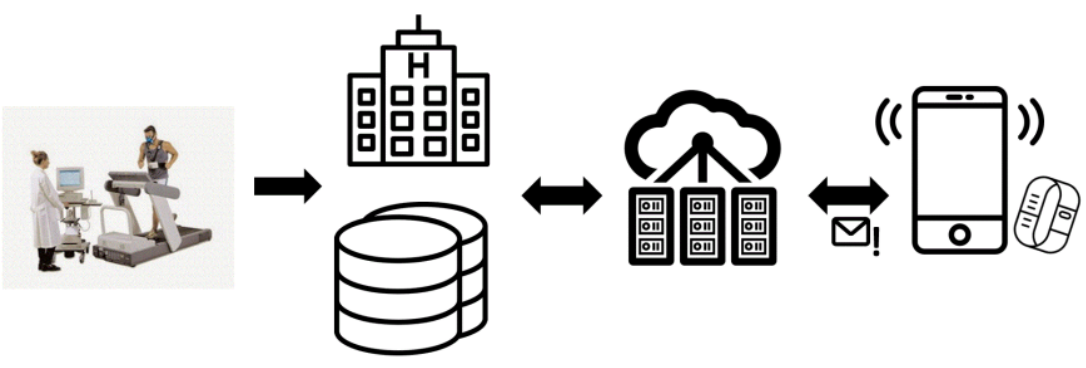

Figure 2

System configuration and data flow. The left sub-figure represents cardio-pulmonary exercise testing

(CPX) assessments. The detected personal CPX testing records is recorded in the database server located in the hospital medical information center (the second sub-figure from left). The rightest sub-

figure shows the wireless health bracelet device for biometric feature detection and a smartphone device for data transfer. The collected biometric features from HF patients are sent to the cloud server for real-time evaluation (the third sub-figure from left). The cloud server also takes responsibility for sending warning messages to appropriate recipients through Short Message Service (SMS). 


\subsection{Construction of Peak Limitations from Standard Reference Curves}

Peak limitations for both MET and HR parameters for different ages and genders were constructed for two healthy male and female groups. These two sets of peak limitation bounding boxes in MET vs. HR measurements were obtained by transforming the original figure of peak $\mathrm{VO}_{2}(\mathrm{~mL} / \mathrm{min})$ vs. age (years) as defined by Fleg et al. [21].

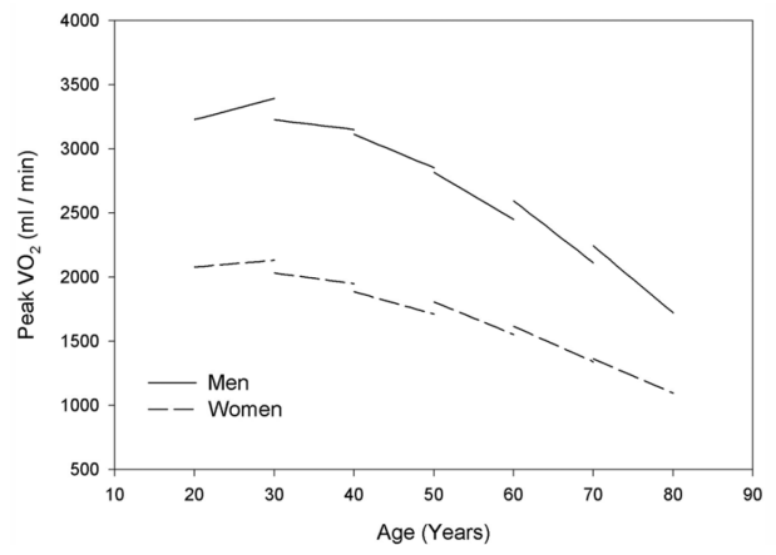

(a)

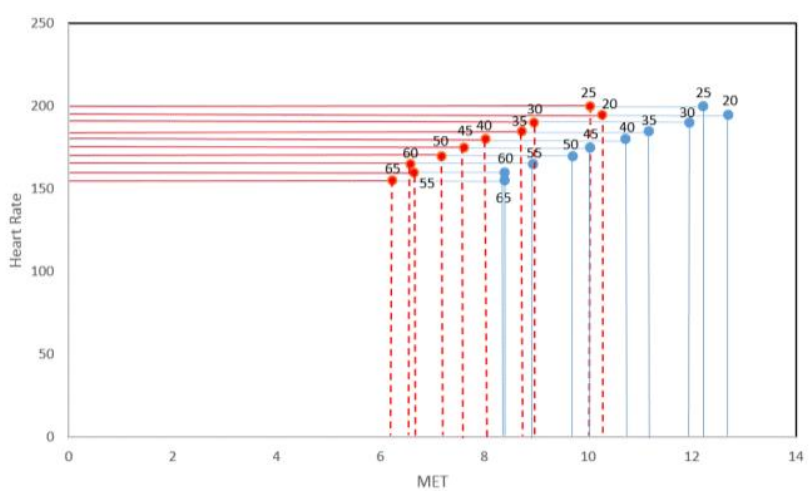

(b)

Figure 3

Standard reference curve transformation and construction for data calibration. (a) Peak $\mathrm{VO}_{2}(\mathrm{~mL} / \mathrm{min})$ vs. age (years) defined by American Heart Association (Reprinted from Fleg et al. [21]); (b)

Transformed standard peak $\mathrm{VO}_{2}$ vs. age to maximum limitation of MET/HR as safety bounding boxes for various ages/gender. Red dotted bounding boxes for female and blue solid bounding boxes for male. Corresponding ages are shown in the upper-right corners. 
The original figure is reprinted in Figure 3(a) and the transformed corresponding boundaries are shown in Figure 3(b). Maximum MET values were obtained by dividing peak $\mathrm{VO}_{2}(\mathrm{~mL} / \mathrm{min})$ by 3.5 and the average weight $(\mathrm{kg})$ of the healthy people, and corresponding HR values were obtained by subtracting age in years from 220. Thus, two sets of limitation bounding boxes for both males and females were obtained as shown in Figure 3(b). It should be noted that each bounding box represents a set of default maximum limitations for HF patients with the same age and gender. Once an HF patient wears the IoMT device for self-exercise training, all collected MET and HR values will be evaluated in a real-time manner and compared to her/his own customized limitations assigned by rehabilitation physicians. If there is no clear definition by a physician, the general rules of setting initial maximum limitations for an HF patient are used with a range of 40$80 \%$ of peak MET and 220 - age in years for HR parameter according to the HF patient's age and gender. Thus, every HF patient will have a customized maximum limitation region for monitoring safety.

\subsection{Exercise Training Statistics for HF Patients}

A total of $1035 \mathrm{HF}$ patients with CPX testing records from the CGMH database were retrieved from 4 divisions. Six attributes including sex, age, weight, maximum heart rate achieved, MET and CPX testing limitations were retrieved from their corresponding medical documents. Only 728 male and 327 female entries were retrieved for analysis, as the CPX testing equipment were limited and only a proportion (1.6\%) of HF patients were qualified and willing to accept exercising training. Because of the general symptoms of dyspnea and fatigue, most HF patients are reluctant to participate in rehabilitation programs, even if they understand that exercise training may postpone rehospitalization and reduce the risk of mortality. This is particularly true for the elderly, who are the major population of HF patients. Only partial distributions of CPX testing results are shown in Figure 4(a). Different clusters represent the conditions of HF patients and healthy people: right green triangle cluster for healthy group with age ranging from 25 to 35 years; middle yellow circle cluster for HF patients with age ranging from 25 to 35 years; left red diamond shape cluster for HF patients with age from 55 to 65 years. Healthy and young groups clearly cluster to the right locations within the MET vs. HR measurements. In this study, the average number of CPX tests conducted was 1.5 times per person among the $1035 \mathrm{HF}$ patients, and the maximum testing record was five. Here, we display a case of an HF patient who attended 5 exercise training in Keelung CGMH, and the trained sequential conditions are shown in Figure 4(b). The patient's maximum MET and HR measurements were clearly, significantly improved. This condition was true for all HF patients. Hence, we suggest that after the stable conditions of HF patients are confirmed by doctors and rehabilitation physicians, the patients should be advised to consistently perform self-training at home. For these cases, the proposed safe mechanism using IoMT bracelets should be considered. 


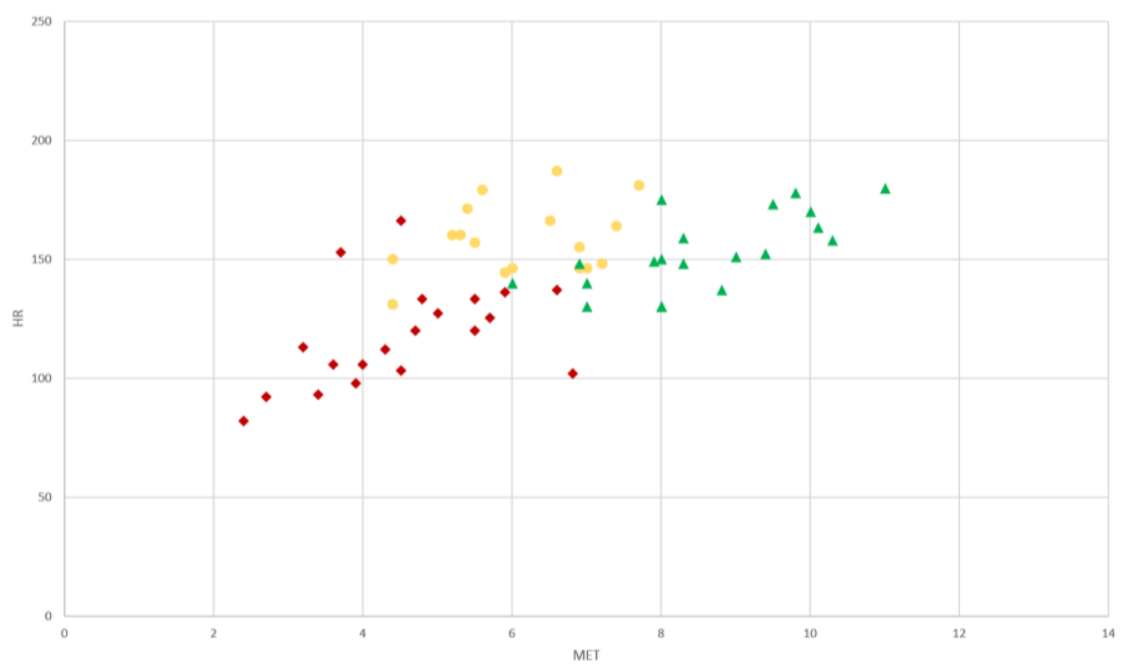

(a)

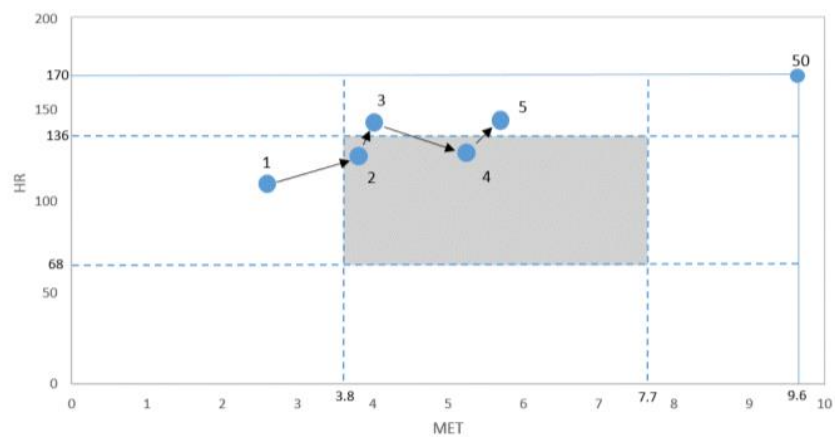

(b)

Figure 4

CPX testing measurements from collected data. (a) Different clusters of HF patients and healthy people: right green triangles for healthy group (age from 25 to 35 years); middle yellow circles for HF patients (age from 25 to 35 years); left red diamond shape dots for HF patients (age from 55 to 65 years); (b). An example HF patient with 5 exercise training data, sequentially. The maximum limitation box (solid lines) derived from healthy people is shown, and the conditions of the patient improved following the

MET/HR measurements and move towards those of healthy people.

\subsection{Warning Functions}

Because of the sudden, urgent, and fatal characteristics of HF patients, the proposed warning mechanism is categorized into three different types. The first warning function is designed for users who have not been previously diagnosed with HF or any cardio-related symptoms. The system provides corresponding 
maximum limitation bounding boxes obtained from a healthy group of people. If the data are collected from a user after exercise training and all data points are relatively lower and distant from the default bounding box, she/he should be very careful about her/his heart conditions. This rough clustering and warning message may help people who are unaware that their heart conditions are already at a highrisk level. The second warning function is designed for HF patients as a secure monitoring mechanism. Based on the patient's exercise training conditions, rehabilitation physicians provide exercise prescription advice regarding maximum limitations of MET and HR references. If patients surpass the restriction levels after any self-training exercises, the server will send an instant warning message to the patient to remind her/him to lower the exercise intensity. The third warning messages are designed for an emergency condition when the user's heartbeat rate suddenly decreases to a predetermined low level; the system will send out an instant message to her/his doctors and family for emergency treatment. This mechanism is designed for serious HF patients who exercise alone.

\section{Conclusions}

Accurate classification of HF types may enable doctors to make better decisions regarding surgical and medication treatments for their HF patients. Here, we analyzed medical documents from non-intrusive ultrasound and X-ray examination records to determine the features of EF values, valvular conditions, edema, congestion, and ICD-9 codes for AF. Using straightforward classification rules, we effectively increased the diagnosis accuracy of HF types for providing proper prognostic healthcare. In addition, evaluating CPX testing performance may assist HF patients in understanding their rehabilitation limitations and function as guidelines for self-exercise training programs at home. To avoid increased cardiovascular mortality risk, particularly when HF patients perform exercise training without rehabilitation professionals aside, a safer mechanism is desired, which is successfully provided in this study. The proposed application system sets parameters for maximum MET and HR measurements either from a rehabilitation physician's exercise prescription or from default settings obtained from controlled healthy groups. A real-time monitoring system retrieves both MET and HR features from users' bracelets and provides an efficient and effective mechanism of sending warning messages to the patients when maximum limitations are exceeded. If the conditions are extremely severe for the HF patient, a warning message will also be sent to their family, doctors and/or caregivers.

According to the distributions of MET/HR features for HF patients, the CPX testing conditions of HF patients are significantly lower than for healthy people, and these clustered MET/HR features can be applied as reference indicators for HF symptom detection. Using appropriate protocols for exercise training programs, HF patients can enhance their heart conditions and improve their quality of life by consistently participating in rehabilitation therapies. This proposed warning mechanism applies not only to HF patients, but also to users who may be unaware of their HF symptoms. Additionally, the application 
mechanism used in this study is applicable for different disease targets, such as, diagnosis, classification and early warning models for various chronic diseases. In summary, we propose a safe system that collects MET and HR values through IoMT bracelets and designed default safety regions of MET vs. HR for HF patients of different age, gender and weight to, monitor self-training programs. We expect to provide users with an effective warning mechanism through bracelet devices and perform automatic detection and prevention for both HF patents and healthy people.

Though the prototype of the proposed warning system is successfully implemented herein, a field experimental test should be applied to verify the performance of the proposed system. A large-scale field experiment project is currently in progress. To assure that appropriate steps are performed, to protect the rights and welfare of humans participating as the subjects in our study, we have raised a research program to the Institutional Review Board (IRB) for approval. When IRB reviews and approves our research program, we will start to randomize all collected subjects into treatment and control groups, and the experimental outcomes and usage behavior between these two groups will be carefully compared. Meanwhile, in addition to the detected features of MET and HR from self-trained patients, we will try to discover possible associations between the measured features and the classified HF types. New biometric features from the IoMT devices will be considered and adopted in this study, if they are effective and stable. According to the confirmed relationship between measurable features and HF groups, we will try to organize the best customized default settings for each individual HF patient.

\section{Acknowledgement}

This work is supported by Ministry of Science and Technology, Taiwan, R.O.C. (MOST 104-2321-B-019-009 to T.-W. Pai and MOST 105-2634-E-019-001 to Lin) and Chang Gung Memorial Hospital, Keelung, Taiwan (CMRPG2C0351 to C.-H. Wang).

\section{References}

[1] O. S. Ogah, S. Stewart, O. E. Onwujekwe, A. O. Falase, S. O. Adebayo, T. Olunuga and K. Sliwa, "Economic Burden of Heart Failure: Investigating Outpatient and Inpatient Costs in Abeokuta, Southwest Nigeria," PLoS One, Vol. 9, No. 11, pp. e113032, 2014

[2] R. L. Page, J. A. Joglar, M. A. Caldwell, H. Calkins, J. B. Conti, B. J. Deal, N. A. Estes, $3^{\text {rd }}$, M. E. Field, Z. D. Goldberger, S. C. Hammill, J. H. Indik, B. D. Lindsay, B. Olshansky, A. M. Russo, W. K. Shen, C. M. Tracy and S. M. Al-Khatib, "2015 ACC/AHA/HRS Guideline for the Management of Adult Patients With Supraventricular Tachycardia: A Report of the American College of Cardiology/American Heart Association Task Force on Clinical Practice Guidelines and the Heart Rhythm Society," J Am Coll Cardiol, Sep 16, 2015 
[3] A.-J. Hsiao, L.-H. Chen, and T.-H. Lu, "Ten Leading Causes of Death in Taiwan: A Comparison of Two Grouping Lists," Journal of the Formosan Medical Association, Vol. 114, Issue 8, pp. 679-680, 2014

[4] L. N. Aminde, A. Dzudie, and A. P. Kengne, "Prevalent Diabetes Mellitus in Patients with Heart Failure and Disease Determinants in Sub-Saharan Africans having Diabetes with Heart Failure: a Protocol for a Systematic Review and Meta-Analysis," BMJ Open, Vol. 6, No. 2, pp. e010097, 2016

[5] P. Ponikowski, A. A. Voors, S. D. Anker, H. Bueno, J. G. F. Cleland, A. J. S. Coats, V. Falk, J. R. González-Juanatey, V.-P. Harjola, E. A. Jankowska, M. Jessup, C. Linde, P. Nihoyannopoulos, J. T. Parissis, B. Pieske, J. P. Riley, G. M. C. Rosano, L. M. Ruilope, F. Ruschitzka, F. H. Rutten, P. Meer, "2016 ESC Guidelines for the Diagnosis and Treatment of Acute and Chronic Heart Failure: The Task Force for the Diagnosis and Treatment of Acute and Chronic Heart Failure of the European Society of Cardiology (ESC) Developed with the special contribution of the Heart Failure Association (HFA) of the ESC." European heart journal, 2016

[6] M. Davies, F. Hobbs, R. Davis, J. Kenkre, A. K. Roalfe, R. Hare, D. Wosornu, R. J. Lancashire, "Prevalence of Left-Ventricular Systolic Dysfunction and Heart Failure in the Echocardiographic Heart of England Screening Study: a Population-based Study," Lancet, Vol. 358, No. 9280, pp. 439-44, 2001

[7] B. A. Borlaug and W. J. Paulus, "Heart Failure with Preserved Ejection Fraction: Pathophysiology, Diagnosis, and Treatment," Eur Heart J, Vol. 32, No. 6, pp. 670-9, 2011

[8] B. Iung, G. Baron, E. G. Butchart, F. Delahaye, C. Gohlke-Barwolf, O. W. Levang, P. Tornos, J. L. Vanoverschelde, F. Vermeer, E. Boersma, P. Ravaud and A. Vahanian, "A Prospective Survey of Patients with Valvular Heart Disease in Europe: The Euro Heart Survey on Valvular Heart Disease," Eur Heart J, Vol. 24, No. 13, pp. 1231-43, Jul, 2003

[9] Z. H. Chen, Y. R. Jiang, J. Q. Peng, J. W. Ding, S. Li, J. Yang, H. Wu and J. Yang, "Clinical Effects of Combined Treatment by Optimal Dose of Furosemide and Spironolactone on Diastolic Heart Failure in Elderly Patients," Exp Ther Med, Vol. 11, No. 3, pp. 890-894, 2016

[10] M. F. Jimenez-Navarro, J. M. Garcia-Pinilla, A. M. Trujillo and E. de Teresa Galvan, "Treatment of Heart Failure due to Systolic Dysfunction," Rev Esp Cardiol, Vol. 6 Suppl. F, pp. 46-52, 2006

[11] A. J. Camm, P. Kirchhof, G.Y.H. Lip, U. Schotten, I. Savelieva, S. Ernst, I. C. Van Gelder, N. Al-Attar, G. Hindricks, B. Prendergast, H. Heidbuchel, O. Alfieri, A. Angelini, D. Atar, P. Colonna, R. De Caterina, J. De Sutter, A. Goette, B. Gorenek, M. Heldal, S. H. Hohloser, P. Kolh, J.-Y. Le Heuzey, P. Ponikowski, F. H. Rutten, "Guidelines for the Management of Atrial Fibrillation: the Task Force for the Management of Atrial Fibrillation of the European Society of Cardiology (ESC) European Heart Journal, 31(19), 2369-429, 2010 
[12] W. M. Mampuya, "Cardiac Rehabilitation Past, Present and Future: An Overview," Cardiovascular Diagnosis and Therapy 2(1), pp. 38-49, 2012

[13] D. Fischer , S. Rossa, U. Landmesser, S. Spiekermann, N. Engberding, B. Hornig, H. Drexler, "Endothelial Dysfunction in Patients with Chronic Heart Failure is Independently Associated with Increased Incidence of Hospitalization, Cardiac Transplantation, or Death," Eur Heart Journal, Vol. 26(1), pp. 65-69, 2005

[14] R. Hambrecht, E. Fiehn, J. Yu, J. Niebauer, C. Weigl, L. Hilbrich, V. Adams, U. Riede, G. Schuler, "Effects of Endurance Training on Mitochondrial Ultrastructure and Fiber Type Distribution in Skeletal Muscle of Patients With Stable Chronic Heart Failure," J Am Coll Cardiol, Vol. 29(5), pp. 1067-1073, 1997

[15] P. J. Mueller, "Exercise Training and Sympathetic Nervous System Activity: Evidence for Physical Activity Dependent Neural Plasticity," Clin Exp Pharmacol Physiol, Vol. 34(4), pp. 377-384, 2007

[16] J. A. Woods, K. R. Wilund, S. A. Martin, and B. M. Kistler, "Exercise, Inflammation and Aging," Aging Dis., Vol. 3(1), pp. 130-140, 2012

[17] G. J. Balady, R. Arena, K. Sietsema, J. Myers, L. Coke, G. F. Fletcher, D. Forman, B. Franklin, M. Guazzi, M. Gulati, S. J. Keteyian, C. J. Lavie, R. Macko, D. Mancini, R. V. Milan, "Clinician's Guide to Cardiopulmonary Exercise Testing in Adults: A Scientific Statement from the American Heart Association,". Circulation, Vol. 122, pp. 191-225, 2010

[18] C. Doukas and I. Maglogiannis, "Bringing IoT and Cloud Computing towards Pervasive Healthcare," IEEE Proceedings of the Sixth International Conference Innovative Mobile and Internet Services in Ubiquitous Computing (IMIS), pp. 922-926, 2012

[19] M. Jette, K. Sidney, G. Blumchen, "Metabolic Equivalents (METS) in Exercise Testing, Exercise Prescription, and Evaluation of Functional Capacity," Clin. Cardiol, Vol. 13, pp. 555-565, 1990

[20] G. F. Fletcher, P. A. Ades, P. Kligfield, R. Arena, G. J. Balady, V. A. Bittner, L. A. Coke, J. L. Fleg, D. E. Forman, T. C. Gerber, M. Gulati, K. Madan, J. Rhodes, P. D. Thompson, M. A. Williams; American Heart Association Exercise, Cardiac Rehabilitation, and Prevention Committee of the Council on Clinical Cardiology, Council on Nutrition, Physical Activity and Metabolism, Council on Cardiovascular and Stroke Nursing, and Council on Epidemiology and Prevention, "Exercise standards for testing and training: a scientific statement from the American Heart Association," Circulation, Vol. 128(8), pp. 873-934, 2013

[21] J. L. Fleg, C. H. Morrell, A. G. Bos, L. J. Brant, L. A. Talbot, J. G. Wright, E. G. Lakatta, "Accelerated Longitudinal Decline of Aerobic Capacity in Healthy Older Adults," Circulation, Vol. 112(5), pp. 674-82, 2005 